\title{
Comunicación Masiva del Ramo Superficial del Nervio Radial con el Nervio Cutáneo Antebraquial Lateral, Implicancias Anatomo-Clínicas. Reporte de un Caso
}

\author{
Massive Communication Between the Superficial Branch of Radial Nerve and Lateral Cutaneous \\ Nerve of the Forearm, Anatomical and Clinical Implications. A Case Report
}

"Martín Inzunza A.; **Guillermo Salgado A.; "***Andrea González S.; ***Juan Carlos De la Cuadra F. \& ** Oscar Inzunza H.

INZUNZA, A. M.; SALGADO, A. G.; GONZÁLEZ, S. A.; DE LA CUADRA, F. J. C. \& INZUNZA, H. O. Comunicación masiva del ramo superficial del nervio radial con el nervio cutáneo antebraquial lateral, implicancias anatomo-clínicas. Reporte de un caso. Int. J. Morphol., 29(3):681-685, 2011.

RESUMEN: La distribución de los ramos nerviosos sensitivos en el borde lateral y en el dorso de la mano han sido descritos con mayor exactitud en las últimas décadas, debido al avance de las técnicas de electrodiagnóstico que ofrecen un alto rendimiento, las cuales han permitido detectar que alrededor del $40 \%$ de la población examinada presenta algún grado de variación anatómica en el territorio de distribución de los nervios involucrados. En este caso presentamos una variación anatómica bilateral extremadamente rara, que involucra al ramo superficial del nervio radial (NRS) y al nervio cutáneo antebraquial lateral (CABL); donde NRS se conecta de forma íntegra con el ramo medial de CABL, formándose así un tronco común (TC) que se distribuye por la región dorsal de la mano. Por su parte, el ramo lateral de CABL se distribuye por el borde lateral de la mano, ocupando el territorio cutáneo de NRS; situación que aparece descrita en la literatura especializada sólo una vez. El hallazgo de estas variaciones anatómicas en los cadáveres disecados con fines docentes en nuestro Departamento de Anatomía, tienen un valor formativo indiscutible para nuestros alumnos de pregrado y especialmente para los de postgrado, quienes pueden comprobar de primera mano la enorme variabilidad del ser humano, valorando las implicancias en la clínica diaria de este conocimiento anatómico.

PALABRAS CLAVE: Nervio radial; Nervio cutáneo lateral antebraquial; Variación anatómica; Inervación; Mano.

\section{INTRODUCCIÓN}

El ramo superficial del nervio radial (NRS) corresponde a un ramo sensitivo que a través de sus tres divisiones: ramo medial (SR1), ramo medio (SR2) y ramo lateral (SR3) inerva la región radial del dorso de la mano y la zona distal del antebrazo (Testut \& Latarjet, 1961; Ikiz \& Üçerler, 2004); describiéndose en estas regiones una sobreproyección con el territorio de distribución del nervio cutáneo antebraquial lateral (CABL), el ramo sensitivo del nervio musculocutáneo (Mackinnon \& Dellon, 1985; Huanmanop et al., 2007; Park et al., 2010).

La situación superficial de NRS y CABL a nivel del tercio distal del radio los expone a ser dañados en procedimientos tales como: la punción venosa de la vena cefálica antebraquial (Boeson et al., 2000), la ejecución de los por- tales radiales para la artroscopía de la articulación radiocarpiana (Ikiz \& Üçerler), la instalación percutánea de fijadores de Kirschner en casos de fracturas distales del radio (Singh et al., 2005) y la instalación negligente de férulas de yeso en el antebrazo (Ikiz \& Üçerler).

Por otro lado, la disposición subcutánea de NRS y CABL permite que estos nervios sean abordados para realizar biopsias nerviosas en el diagnóstico de neuropatía periférica (Chentanez et al., 2010) o para ser utilizados como injerto en la reparación de nervios periféricos (Huanmanop et al.; Wongkerdsook et al., 2011).

En este caso presentamos una variación anatómica bilateral extremadamente rara, que involucra a NRS y CABL,

* Alumno de Medicina, Pontificia Universidad Católica de Chile

** Departamento de Anatomía, Pontificia Universidad Católica de Chile.

**** División de Anestesiología, Escuela de Medicina, Pontificia Universidad Católica de Chile. 
donde el territorio cutáneo de SR3 es tomado por la división lateral de CABL y los ramos SR1 y SR2 surgen de la comunicación entre el ramo medial de CABL y NRS; situación que aparece descrita en la literatura especializada sólo una vez (Huanmanop et al.).

El hallazgo de estas variaciones anatómicas en cadáveres humanos utilizados con fines docentes en nuestro Departamento de Anatomía resulta especialmente atractivo y motivador para nuestros alumnos; quienes logran aquilatar así el valor de la disección anatómica como el método ineludible para aprender, realmente, anatomía humana (Bravo \& Inzunza, 1995). Para los docentes, la comunicación de estos hallazgos nos permite realizar un aporte al conocimiento de las variaciones anatómicas y sus implicancias en la clínica médica (Inzunza \& Burdiles, 2010a, 2010b; Inzunza et al., 2011).

\section{MATERIAL Y MÉTODO}

En un cadáver de sexo masculino, de 82 años de edad, cuya causa de muerte fue sepsis urinaria, que no presentaba lesiones en los miembros superiores, utilizado para fines docentes en nuestro Departamento de Anatomía, se procedió a realizar bajo lupa $3 \mathrm{X}$ la disección bilateral del antebrazo y la mano. Se levantó la piel y se expuso el plano subcutáneo de estas regiones, para seguir el trayecto y el patrón de ramificación de NRS y de CABL; cuidando de visualizar las comunicaciones y el patrón de ramificación que exhibían estos dos nervios. Luego, con la ayuda de un pie de metro digital, se establecieron las dimensiones en $\mathrm{mm}$ de CABL y de NRS. Además, tomando como referencia el proceso estiloides del radio se precisó el punto de convergencia que presentaban estos ramos nerviosos.

\section{RESULTADOS}

Al levantar la piel del tercio distal del antebrazo, en ambas antímeras, se aprecia en el tejido subcutáneo que CABL se divide en una ramo lateral y otro medial a 107,97 $\mathrm{mm}$ del proceso estiloides. El ramo lateral, de 2,45 mm de diámetro, continúa su trayecto hacia distal, pasando sobre la tabaquera anatómica, para abordar el borde radial de la mano, distribuyéndose según lo descrito para la división SR3 de NRS. Por su parte, el ramo medial de CABL, de 2,34 mm de diámetro, luego de un corto trayecto de $26,36 \mathrm{~mm}$ se une con
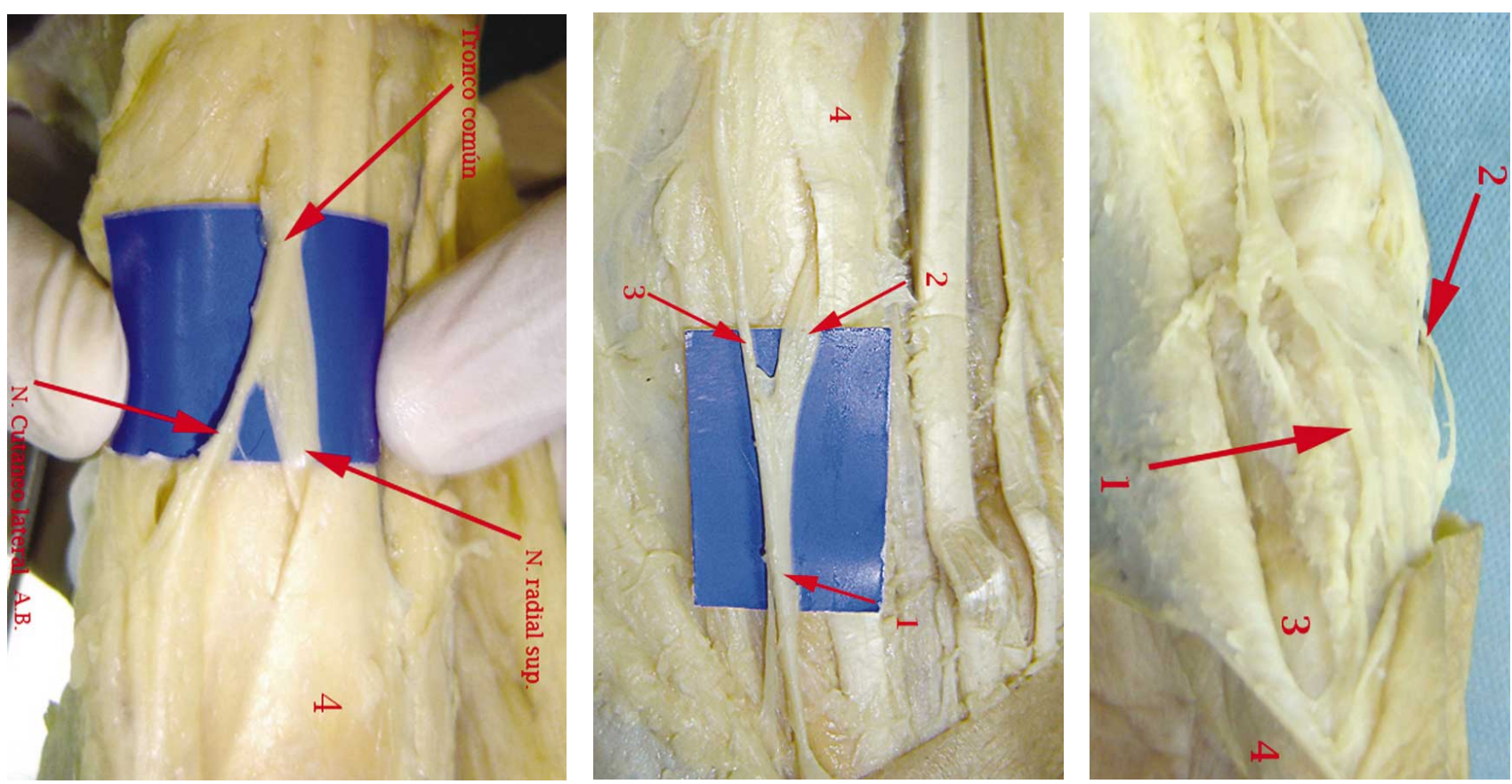

Fig. 1a. Foto que muestra la disposición de los elementos nerviosos en la antímera izquierda. Se observa la comunicación entre NRS y CABL que da origen a TC. 4= Músculo braquiorradial. Fig. 1b: Foto que muestra la disposición de los elementos nerviosos en la antímera derecha. Se observa una situación similar a lo mostrado en la Fig. 1a. 1= TC; 2= NRS; 3= división medial de CABL; 4= Músculo braquiorradial.

Fig. 2. Foto que muestra la disposición de la división lateral de CABL (1), que sigue la distribución cutánea de SR3 (ramo lateral del nervio NRS). 2= Ramo de TC que sigue el territorio de distribución de SR2 (ramo medio de NRS). 3= Cuerpo del primer metacarpiano. 4= Eminencia tenar. 
NRS, formando así un tronco común (TC) que se divide más hacia distal en dos ramos que se distribuyen según lo descrito para los ramos SR1 y SR2 de NRS. El punto de unión entre NRS y CABL se verifica a $72 \mathrm{~mm}$ del proceso estiloides (Figs. 1a y 1b). La disección revela que en ambas antímeras NRS emerge por debajo del tendón del músculo braquioradial, no emite ningún ramo, y se comunica (anastomosa) de manera íntegra con la división medial de CABL.

Los ramos SR1 y SR2, que surgen en este caso de $\mathrm{TC}$, abordan el dorso de la mano distribuyéndose por la piel del segundo y primer espacio metacarpiano respectivamente. Así, SR1 se divide en dos ramos digitales dorsales, uno medial para el tercer dedo y otra lateral para el segundo dedo. Por su parte, SR2 se divide en dos ramos digitales, uno medial para el segundo dedo y otro lateral que sigue hacia el primer dedo, formando el nervio digital dorsal medial del pulgar. A su vez, SR3, que en este caso corresponde a la porción terminal del ramo lateral de CABL, transita por el borde lateral de la mano, emite finos ramos que se extienden 13,08 $\mathrm{mm}$ hacia la región palmar a nivel de la cabeza proximal del primer metacarpiano y $8,37 \mathrm{~mm}$ hacia la region palmar a nivel del borde radial de la diáfisis del mismo, distribuyéndose así por el creciente radial de la región tenar. Además, desde este ramo SR3, originado de CABL, surge el nervio digital dorsal lateral del dedo pulgar (Figs. 2 y 3 ).

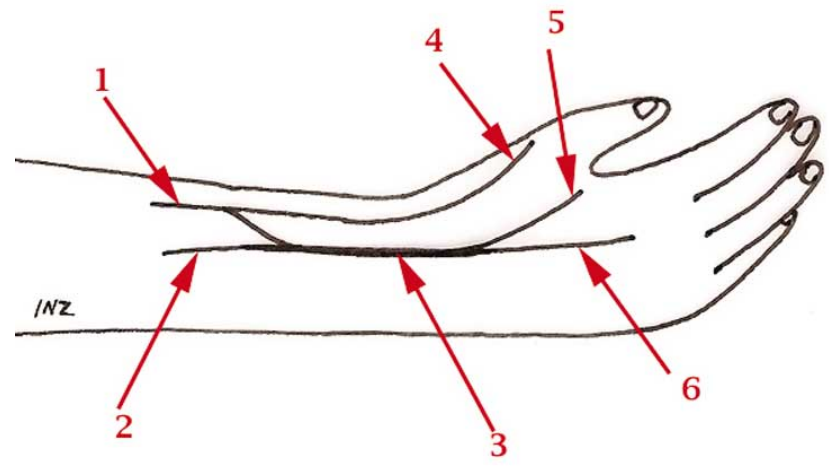

Figura 3: Esquema que resume los hallazgos de la disección. 1= CABL, que se divide en un ramo lateral (4) y en un ramo medial que se une a NRS (2) para formar TC (3). Este nervio variante TC (3) se divide en un ramo medial (6), que sigue el territorio de distribución de SR1 (ramo medial de NRS) y en un ramo lateral (5), que sigue el territorio de distribución de SR2 (ramo lateral de NRS). El ramo lateral de CABL (4) sigue el territorio de distribución de SR3 (que correspondería al ramo lateral de NRS).

\section{DISCUSIÓN}

El patrón general de inervación del antebrazo y la mano ha sido extensamente descrito en diversos textos de anatomía (Testut \& Latarjet; Rouvière \& Delmas, 1984; Moore, 1980; Clemente, 1985). Sin embargo, avances tecnológicos como la artroscopía de la articulación radiocarpiana, la fijación percutánea de fracturas de la mano y del antebrazo y la microcirugía de nervios periféricos, han impulsado a los anatomistas y a los clínicos a revisitar estos temas desde la perspectiva de la anatomía aplicada (Park et al.; Huanmanop et al.; Ikiz \& Üçerler; Marx et al., 2010a, 2010b; Campero et al., 2005). En este proceso de revisión intencionada de la distribución periférica de los nervios de la mano se ha demostrado una gran variabilidad de disposición de estos elementos que, a todas luces, indica que el tema no está agotado.

En efecto, en una investigación clínica de NRS utilizando microneurografía Campero et al. describen tres patrones de distribución cutánea de este nervio, descubriendo una amplia variabilidad entre los pacientes estudiados. De manera interesante, estos autores detectan en un paciente un fascículo de NRS que invade la región palmar, inervando el creciente radial de la eminencia tenar, tal cual lo observamos nosotros en esta disección cadavérica, sólo que en nuestro caso este ramo (SR3) proviene de la división lateral de CABL (Figs. 2 y 3 ).

Numerosos trabajos señalan que los ramos terminales de NRS y CABL establecen conexiones a nivel del borde radial del tercio distal del antebrazo. Esta situación presenta una amplia variabilidad que, según el autor, tiene una incidencia entre el 70\% (Mackinnon \& Dellon) y el 10\% (Tryfonidis et al., 2004). Sin embargo, no existen reportes en la literatura especializada de una comunicación total entre NRS y CABL que, a través de un tronco común, de origen a SR1 y SR2, tal como la encontramos bilateralmente en este estudio (Figs. 1a y 1b).

Si bien las comunicaciones entre NRS y CABL involucran a SR3 y su territorio de distribución (Huanmanop et al.; Ikiz \& Üçerler), sólo existe un reporte en la literatura que demuestra que el territorio de distribución descrito para la división SR3 del nervio NRS es tomado por CABL. Precisamente, Huanmanop et al. encuentran esta situación en dos cadáveres de mujeres tailandesas; patrón de ramificación que se presenta de manera asimétrica en su casuística, y no de disposición simétrica ni en un cadáver de sexo masculino como en nuestro caso (Figs. 1, 2 y 3). Entonces, parece ser que las conexiones entre NRS y CABL aparecen con cierta frecuencia y en varios grupos raciales (Mackinnon \& Dellon; Tryfonidis et al.; Huanmanop et al.; Ikiz \& Üçerler); presentándose estas conexiones en un porcentaje importante de los casos, del orden del $43 \%$, de manera asimétrica (Huanmanop et al.). Sin embargo, los casos donde el territorio SR3 es tomado sólo por CABL son extremadamente raros. 
El fenómeno de invasión de CABL hacia el borde radial de la mano es bastante particular y llama la atención que en caso de ausencia de NRS es el nervio musculocutáneo, a través de CABL, el que inerva ese territorio (Spinner, 1978). Del mismo modo, la invasión de CABL, a través de su ramo lateral, que sigue la disposición de SR3 y se distribuye por el creciente radial de la eminencia tenar es rara y se condice con la situación de agenesia del nervio musculocutáneo, donde el nervio mediano toma su territorio motor y sensitivo de distribución (Prasada Rao \& Chaudhary, 2001). Tomando estos datos en consideración, es posible deducir que, en lo que atañe a la inervación cutánea, es más precisa y estable la disposición de los dermatomas que la situación de los nervios que se distribuyen por una región determinada. Es decir, en este caso, la inervación de la piel del pulgar, tanto en su aspecto dorsal como palmar, es tomada por C6 (Moore; Snell, 2006) ya sea por medio de NRS o CABL o incluso a través del nervio mediano; en consecuencia, el dermatoma es el que manda.

En el sistema nervioso periférico se observan convergencias nerviosas masivas, situación que involucra a nervios mixtos, motores y sensitivos, tal como ocurre en la formación del plexo braquial, el plexo lumbar y el plexo sacro (Moore; Testut \& Latarjet). En el miembro superior la convergencia nerviosa más distal se detecta en la formación del nervio mediano, a nivel de la axila, donde se encuentran su raíz medial y lateral, ambas de carácter mixto; desde aquí hacia distal, todos los nervios plexo braquial se van dividiendo progresivamente. En nuestro caso, describimos una convergencia nerviosa que involucra a ramos sensitivos, NRS y CABL, que forman a TC; disposición que se ubica en el tercio distal de antebrazo. En el miembro inferior se observa normalmente una situación similar, convergencia de ramos sensitivos, en la formación del nervio sural, a nivel de la cara posterior del tercio superior de la pierna (Moore; Testut \& Latarjet).

Por último, el hallazgo de estas variaciones anatómicas en los cadáveres disecados con fines docentes en nuestro Departamento de Anatomía, tienen un valor formativo indiscutible para nuestros alumnos de pregrado, quienes pueden comprobar de primera mano la enorme variabilidad del ser humano, desterrando de sus mentes el arquetipo irreal y ficticio que presentan los modelos anatómicos, herramientas docentes de amplio uso hoy en día en las distintas escuelas de medicina del país. Asimismo, estas observaciones son atractivas para nuestros alumnos de postgrado (becados de anestesia, traumatología, etc.) quienes, por su nivel de formación, pueden justipreciar las implicancias en la clínica diaria de este conocimiento anatómico (Klezl et al., 2001). Además, nos permiten realizar un aporte al conocimiento de las variaciones anatómicas y sus implicancias en la clínica médica (Inzunza \& Burdiles, 2010a, 2010b; Inzunza et al.).

INZUNZA, A. M.; SALGADO, A. G.; GONZÁLEZ, S. A.; DE LA CUADRA, F. J. C. \& INZUNZA, H. O. Massive Communication between the superficial branch of radial nerve and lateral cutaneous nerve of the forearm, anatomical and clinical implications. A case report. Int. J. Morphol., 29(3):681-685, 2011.

SUMMARY: The distribution of the sensory nerve branches in the lateral and the back of the hand have been described more accurately in the past decades due to advancement of high performance electro-diagnostic variation techniques, which indicate that approximately $40 \%$ of the population examined have some degree of anatomical variation in the distribution area of the nerves involved. In this case we present an extremely rare, bilaterally detected variation, involving the superficial branch of the radial nerve (SBRN) and lateral antebrachial cutaneous nerve of the forearm (LABCN), where the SBRN is connected integrally with the medial branch of $\mathrm{LABCN}$, forming a common trunk (CT) distributed by the dorsal region of the hand. Furthermore, the lateral branch of the LABCN is distributed in the lateral border of the hand, occupying the area of the skin of the SBRN, an event that is described only once in the literature. The discovery of these anatomical variations in dissected cadavers for teaching purposes, in the Department of Anatomy, have an undeniable educational value for our undergraduate students and especially for the graduate who can observe the enormous variability of human beings first hand, and value implications of this anatomical knowledge in daily clinic.

KEY WORDS: Radial nerve; Antebraquial cutaneous nerve; Anatomical variation; Innervation; Hand.

\section{REFERENCIAS BIBLIOGRÁFICAS}

Boeson, M. B.; Hranchook, A. \& Stoller, J. Peripheral nerve injury from intravenous cannulation: a case report. AANA J., 68(1):53-7, 2000.

Bravo, H. \& Inzunza, O. Evaluación de algunos programas computacionales en la enseñanza de Anatomía y
Neuroanatomía de la Facultad de Medicina de la Pontificia Universidad Católica de Chile. Rev. Chil. Anat., 13(1):7986, 1995.

Campero, M.; Serra, J. \& Ochoa J. L.; Peripheral projections of sensory fascicles in the human superficial radial nerve. 
INZUNZA, A. M.; SALGADO, A. G.; GONZÁLEZ, S. A.; DE LA CUADRA, F. J. C. \& INZUNZA, H. O. Comunicación masiva del ramo superficial del nervio radial con el nervio cutáneo antebraquial lateral, implicancias anatomo-clínicas. Reporte de un caso. Int. J. Morphol., 29(3):681-685, 2011.

Brain, 128(Pt 4):892-5, 2005.

Chentanez, V.; Agthong, S.; Huanmanop, T.; Pairoh, S. \& Kaewsema, A. Morphometric analysis of the human superficial radial nerve. Anat. Sci. Int., 85(3):167-70, 2010.

Clemente, C. D. Gray's Anatomy. 30 $30^{\text {th }}$ Edn. Philadelphia, Lea \& Febiger, 1985.

Fregnani, J. H.; Macéa, M. I.; Pereira, C. S.; Barros, M. D. \& Macéa, J. R. Absence of the musculocutaneous nerve: a rare anatomical variation with possible clinical-surgical Implications. São Paulo Med. J., 126(5):288-90, 2008.

Huanmanop, T.; Agthong, S.; Luengchawapong, K.; Sasiwongpakdee, T.; Burapasomboon, P. \& Chentanez, $\mathrm{V}$. Anatomic characteristics and surgical implications of the superficial radial nerve. J. Med. Assoc. Thai., 90(7):1423-9, 2007.

Ikiz, Z. A. \& Uçerler, H. Anatomic characteristics and clinical importance of the superficial branch of the radial nerve. Surg. Radiol. Anat., 26(6):453-8, 2004.

Inzunza, O. \& Burdiles, A. Arteria Subclavia Aberrante. Int. J. Morphol., 28(4):1215-9, 2010a.

Inzunza, O. \& Burdiles, A. Arteria Vertebral Izquierda Aberrante. Int. J. Morphol., 28(1):209-12, 2010 b.

Inzunza, O.; Inzunza, M. \& Salgado, G. Vena Renal Izquierda recurrente retroaórtica. Reporte de una rara anomalía. Int. J. Morphol., 29(2):339-43, 2011.

Klezl, Z.; Krejca, M. \& Simcik, J. Role of Sensory Innervation Variations for Wrist Block Anesthesia. Arch. Med. Res., 32(2):155-8, 2001.

Mackinnon, S. E. \& Dellon, A. L. The overlap pattern of the lateral antebrachial cutaneous nerve and the superficial branch of the radial nerve. J. Hand. Surg. Am., 10(4):5226, 1985.

Marx, S. C.; Kumar, P.; Dhalapathy, S.; Marx, C. A.; Babu, M. S. \& Bhat, K. M. Histological and ultrasonographical study of the human superficial branch of the radial nerve at distal forearm and its clinical implications. Rom. J. Morphol. Embryol., 51(4):751-8, 2010a.

Marx, C.; Kumar, P.; Dhalapathy, S. Prasad, K.; Marx, A. Microanatomical and immunohistochemical study of the human lateral antebrachial cutaneous nerve of forearm at the antecubital fossa and its clinical implications. Clin. Anat. 23:693-701, 2010b.
Moore, K. Clinically oriented Anatomy. Baltimore, Williams \& Wilkins, 1980.

Park, B. K.; Bun, H. R.; Hwang M.; Hong, J. \& Kim, D. H. Medial and lateral branches of the superficial radial nerve: Cadaver and nerve conduction Studies. Clin. Neurophysiol., 121(2):228-32, 2010

Prasada Rao, P. V. \& Chaudhary, S. C. Absence of musculocutaneous nerve: two case reports. Clin. Anat., 14(1):31-5, 2001.

Rouvière, H. \& Delmas, A. Anatomía Humana Descriptiva, Topográfica y Funcional. $11^{a}$ Ed. Barcelona, Masson, 2005. Tomo 3.

Singh, S.; Trikha, P. \& Twyman, R. Superficial radial nerve damage due to Kirschner wiring of the radius. Injury, 36(2):330-2, 2005.

Snell, R. S. Neuroanatomía Clínica. $5^{\mathrm{a}}$ edn. Buenos Aires, Editorial Médica Panamericana, 2005.

Spinner, M. Injuries to the major branches of Peripheral nerves of the forearm. $2^{\text {nd }}$ edn. Philadelphia, Saunders, 1978. pp.137-8.

Testut, L. \& Latarjet, A. Tratado de Anatomía Humana. $9^{\mathrm{a}}$ Edn. Barcelona, Salvat Editores S. A., 1961.

Tryfonidis, M.; Jass, G. K.; Charalambous, C. P. \& Jacob, S. Superficial branch of the radial nerve piercing the brachioradialis tendon to become subcutaneous: an anatomical variation with clinical relevance. Hand Surg., 9(2):191-5, 2004.

Wongkerdsook, W.; Agthong, S.; Amarase, C.; Yotnuengnit, P.; Huanmanop, T. \& Chentanez, V. Anatomy of the lateral antebrachial cutaneous nerve in relation to the lateral epicondyle and cephalic vein. Clin. Anat., 24(1):56-61, 2011.

Dirección para correspondencia:

Prof. Dr. Oscar Inzunza

Departamento de Anatomía Normal

Escuela de Medicina

Pontificia Universidad Católica de Chile

CHILE

Email: oinzunza@med.puc.cl
Recibido : 02-05-2011

Aceptado: 14-06-2011 